\title{
Signs of increased leakage over the jejunal mucosa during gliadin challenge of patients with coeliac disease
}

\author{
B Lavö, L Knutson, L Lööf, B Odlind, R Hällgren
}

\begin{abstract}
Intestinal secretion rates of albumin, hyaluronan, and beta $a_{2}$-microglobulin (beta ${ }_{2}$ micro) were determined under basal conditions and after gliadin challenge of coeliac patients and healthy controls by the use of a jejunal perfusion technique. A new tube system was used where a jejunal segment is isolated between balloons and then perfused with a balanced salt solution. Under basal conditions the secretion rate of albumin was similar in the patients and controls while the secretion rate of the glycosaminoglycan hyaluronan, a high molecular weight connective tissue component, was increased more than two times in coeliac patients. Beta ${ }_{2}$-micro was secreted in on average three-fold rates in coeliacs compared with controls. All three substances were secreted at a higher rate in patients with active disease than in those with inactive disease defined by morphological damage in small bowel biopsies. The concentrations in jejunal perfusion fluids relative to serum levels in the coeliac patients were for albumin $\mathbf{0 \cdot 0 0 0 7}$, beta - micro $0 \cdot 10$, and for hyaluronan 1.94. Challenge with a single dose of gliadin into the jejunal segment gave within 60 min a significant, about two-fold, increase of the secretion rates of all three measured substances. The appearance of hyaluronan could reflect a gliadin induced mucosal oedema with an enhanced leakage from the interstitial/lymph fluid, rich in this glycosaminoglycan. The observed parallel increases in the jejunal secretion of albumin and beta ${ }^{-}$ micro after gliadin challenge are best explained by a similar mechanism.
\end{abstract}

Department of Internal Medicine, University Mospital, Uppsala B Lavö

L Lööf

R Hällgren

Department of Surgery, University Hospital, Uppsala

L Knutson

Department of Research and Development, Pharmacia AB, Uppsala, Sweden

Sweden

Correspondence to: Roger Hällgren MD, Department of Internal Medicine University Hospital, S-751 85 Uppsala, Hospital,

Accepted for publication 10 May 1989
In coeliac disease the small bowel mucosa of genetically predisposed individuals is damaged after ingestion of wheat gluten and similar proteins in rye and barley. The pathophysiological events that leads to the characteristic 'flat' mucosa with damaged enterocytes and infiltration of inflammatory cells into the epithelium and lamina propria $^{1-3}$ have not been fully elucidated. The proposed mechanisms for the mucosal damage have been primarily immunological including both reactions elicited by antigen-antibody complexes and cell mediated damage. $^{2-6}$ It has been reported that gliadin challenge may induce histological changes of the mucosa as early as within two hours after gliadin administration. ${ }^{+6}$ Studies of the actue pathological events induced by gliadin challenge, however, have been hampered by the difficulties in obtaining serial samples from the mucosa.
Inflammatory affection of the mucosa might influence the leakage from the capillary bed/ interstitial fluid to the bowel lumen. The aim of this study was to elucidate this possibility by continuous measurements of the intestinal appearance of substances from plasma and/or lymph fluid under basal conditions and after local gliadin challenge of a perfused jejunal segment. This was made possible by the use of a recently developed multichannel tube with two occluding balloons allowing measurement of the secretion in an isolated small bowel segment. ' In this study we report the jejunal appearance rates of low and high molecular weight substances; albumin, beta $a_{2}$-microglobulin (beta $a_{2}$-micro), and hyaluronan. The appearance of albumin in the intestinal perfusion fluid should merely reflect the protein leakage from the blood circulation and the intestinal interstitial fluid. Hyaluronan, a high molecular weight glycosaminoglycan, is an important connective tissue component synthetised mainly by mesenchymal cells and present in virtually every tissue of the body. ${ }^{8}$ It is drained from tissues by the lymph and appears in the lymph fluid in concentrations much higher than in plasma. ${ }^{89}$ Thus, the appearance of hyaluronan in intestinal fluids might reflect a local synthesis in the intestinal wall and a leakage from, primarily, the lymph or interstitial fluid. The jejunal appearance of the low molecular weight protein beta ${ }_{2}$-micro should also reflect a passive leakage but, in addition, it might as well reflect a local intestinal production, since this protein is part of the HLA class I receptors present on all nucleated cells. An increased production of beta ${ }_{2}$-micro can therefore be anticipated during an increased turnover of cells. ${ }^{10}$

\section{Methods}

\section{PATIENTS}

Ten patients with coeliac disease were studied by the jejunal perfusion technique. They were a selection of treated patients with a good clinical response to a gluten free diet and patients with a bad response because of dietary negligence. Two untreated patients were also studied; they later responded favourably to a gluten free diet and have normalised their small bowel mucosa. Seven of the patients were given a local challenge with gliadin into the jejunal segment. One patient was investigated twice, with and without gliadin challenge. Data concerning age, sex, disease duration, serum gliadin antibody level, dietary status, and histopathological findings are given in Table I. Healthy controls $(n=14)$ were 
TABLE I Clinical data on patients with coeliac disease investigated by regional jejunal perfusion under basal conditions and after gliadin challenge

\begin{tabular}{|c|c|c|c|c|c|c|c|}
\hline Patient & Age & Sex & $\begin{array}{l}\text { Year of } \\
\text { diagnosis }\end{array}$ & Diet & $A G A$ & $\begin{array}{l}\text { Small } \\
\text { bowel } \\
\text { histology }\end{array}$ & $\begin{array}{l}\text { Gliadin } \\
\text { challenge }\end{array}$ \\
\hline & 66 & $F$ & 1981 & GFD & 59 & PVA & - \\
\hline DA & 51 & M & 1987 & & 350 & PVA & - \\
\hline & 41 & $M$ & 1977 & GFD & 17 & $\mathrm{~N}$ & - \\
\hline & 71 & M & 1960 & GFD & 70 & PVA & - \\
\hline & 43 & $M$ & 1987 & $\mathrm{~N}$ & 300 & SVA & + \\
\hline VS & 60 & $\mathrm{~F}$ & 1979 & GFD & 200 & PVA & + \\
\hline $\mathrm{KJ}$ & 33 & $F$ & 1986 & GFD & 15 & PVA & + \\
\hline VÖ & 42 & M & 1980 & GFD & 70 & PVA & + \\
\hline OP & 57 & M & 1985 & GFD & 60 & PVA & + \\
\hline ES & 40 & $\mathrm{~F}$ & 1985 & GFD & 7 & $\mathrm{~N}$ & + \\
\hline $\mathrm{CL}$ & 41 & M & 1977 & GFD & 17 & $\mathbf{N}$ & + \\
\hline
\end{tabular}

Abbreviations: Diet; $\mathrm{GFD}=$ gluten free diet, $\mathrm{N}=$ normal diet. AGA = gliadin antibody titre (IgA; normal values of healthy $<48$ units). Histology of duodenal biopsy; SVA = subtotal villous atrophy, PVA = partial villous atrophy, $\mathrm{N}=$ normal villous architecture. Gliadin challenge $-=$ no gliadin, $+=$ gliadin

also investigated with jejunal perfusion; three were challenged with gliadin under conditions identical to those for the coeliac patients. The mean age of the controls was 27 years (range 22$32)$.

\section{SEGMENTAL JEJUNAL PERFUSION}

Perfusions were performed after an overnight fast as described elsewhere. ${ }^{7}$ In brief, we used a six lumen polyvinyl chloride tube with an external diameter of $5.3 \mathrm{~mm}$ (16 French) (Fig 1). The distal end of the tube contains a tungsten weight attached to facilitate passage of the tube to the jejunum. Proximal to the weight there are two inflatable latex balloons placed $10 \mathrm{~cm}$ apart. The segment between the balloons is connected with two wide channels allowing infusion and evacuation of perfusion fluids. Proximal to the segment, there are smaller peripheral channels for administration of marker substances and drainage.

The tube was introduced orally after local anaesthesia with lignocaine. A Teflon coated guide wire was used during insertion of the tube. The tube was positioned with fluoroscopic control, with the segment for perfusion in the proximal jejunum. Air was inflated into the balloons and the isolated segment was rinsed with isotonic saline. The cleaning of the segment Gastric flutd is drained by a
separate tube. Phenol red is the marker for proximal leakage and ${ }^{+1} \mathrm{C}-\mathrm{PEG} 4000$ a marker of perfusion fluid recovery. was continued for 30 minutes or until the recovered fluid was visually clean. The volume of the isolated bowel segment was experimentally found to be $80-100 \mathrm{ml}$. A separate standard gastric tube was positioned in the stomach for continuous drainage of gastric secretions through suction. After the rinsing period, the segment was perfused at $3 \mathrm{ml} / \mathrm{min}$ with a solution containing $10 \mathrm{mmol}$ glucose, $5.4 \mathrm{mmol} \mathrm{KCl}, 120$ mmol NaCl, 2 mmol Na2HPO4, 1 g/l polyethylene glycol (PEG) 4000, and $35 \mathrm{mmol}$ mannitol. ${ }^{14} \mathrm{C}$-labelled PEG (Mw 4000 daltons, $2.5 \mu \mathrm{Ci} / \mathrm{l}$; Amersham, Buckinghamshire, England) was added to the perfusion fluid as a volume marker. Aprotinin $(10000 \mathrm{KIU} / \mathrm{ml}$; Bayer AG, Leverkusen FRG) was added to the perfusion fluid, $10 \mathrm{ml} / \mathrm{l}$, to inhibit possible proteolytic activity in the perfused segment. Phenol red solution ( $50 \mathrm{mg} / \mathrm{l}$ saline) was infused proximally to the first balloon at a rate of $1 \mathrm{ml}$ min and analysed in the effluent from the intestinal segment. All solutions infused were $37^{\circ} \mathrm{C}$. The jejunal perfusion fluid was collected by gravity drainage. The perfusion fluids were collected on ice and were fractionated in $20 \mathrm{~min}$ samples. The fluids were frozen at $-70^{\circ} \mathrm{C}$ in small samples of 1-2 ml pending analysis. The patients were recumbent during the whole perfusion period of 160-180 min. All patients were examined after an overnight fast. The mean recovery of ${ }^{14} \mathrm{C}$-PEG 4000 during perfusion was $94 \%$, range $83-100$. The mean leakage of phenol red to the segment was $<2 \%$, range $<1-3$.

\section{GLIADIN CHALLENGE}

Gliadin (crude gliadin, Sigma Chemical Co, St Louis, Mo), 15 (4) mg (SEM) was dissolved in $0.5 \mathrm{ml} 70 \%$ ethanol and then mixed into $30 \mathrm{ml}$ of the perfusion fluid. After a $40-60 \mathrm{~min}$ basal perfusion, gliadin was administered as an infusion to the segment during 20 minutes. After gliadin administration the perfusion was continued for another $120 \mathrm{~min}$. The gliadin concentration in the perfused segment was calculated to be $0.2 \mathrm{mg} / \mathrm{ml}$. Concentrations in that range have been found to give a reaction in cell culture experiments. ${ }^{11}$

ANALYTICAL MEASUREMENTS

${ }^{1+} \mathrm{C}$-PEG was determined by liquid scintillation counting (LKB Rackbeta II, Wallac Oy, Turku, Finland) and $1 \mathrm{ml}$ aliquots in duplicate for 15 minutes. ${ }^{12}$ Phenol red was measured spectrophotometrically (Hitachi spectrophotometer model 101, Hitachi Ltd, Tokyo, Japan) at 520 $\mathrm{nm}$ after alkalinisation. ${ }^{13}$

In order to further assure that contamination of the effluent from pancreatic secretion did not occur, the trypsin content of the effluent was measured, using N-p-tosyl-L-arginine methylesther (TAME) as substrate, and determined spectrophotometrically at $247 \mathrm{~nm}^{1+}$ The total proteolytic activity of the effluent was also assessed using casein as substrate. ${ }^{15}$ Before analysis the samples were thawed on ice and $2 \mathrm{mmol}$ phenylmethylsulphonyl fluoride (PMSF; Sigma Chemical Co), a serine protease inhibitor, was added to avoid influences of 
minute amounts of proteases in the effluent.

The perfusion fluid samples were analysed in sequence and in duplicate for the content of albumin, hyaluronan and beta $a_{2}$-microglobulin. Hyaluronan was analysed according to the principles previously outlined. ${ }^{16}{ }^{17}$ In this study, a modified technique was used as developed by Pharmacia Diagnostics (Uppsala, Sweden). Both of the tests are based on the use of specific hyaluronan binding proteins (HABP) isolated from bovine cartilage. In the modified test, the hyaluronan from the samples $(100 \mu \mathrm{l})$ is allowed to bind ${ }^{125}$ I-labelled hyaluronan binding proteins in solution for at least $60 \mathrm{~min}$. The unbound ${ }^{125} \mathrm{I}$ labelled hyaluronan binding proteins is then quantified by incubating with hyaluronan covalently coupled to Sepharose particles. After centrifugation and decanting, the radioactivity bound to the particles is measured. The two techniques give identical results. Albumin in the perfusion fluid (50 $\mu \mathrm{l}$ sample) and beta ${ }_{2}$ microglobulin ( $200 \mu \mathrm{l}$ sample) were measured by double antibody radioimmunoassays (Pharmacia Diagnostics). Parallel standard curves were obtained for all substances by means of the respective standard curves mixed with either buffer or a constant volume of lavage fluid. The variability was less than $8 \%$ for all methods.

STATISTICAL ANALYSIS

Analyses of significance were performed by the use of Student's $t$-test on groups and paired values. The results are expressed both as jejunal fluid concentrations and appearance rates. The appearance rate was based on the steady infusion rate and calculated according to the formula:

concentration in perfusion fluid $\times 3 \mathrm{ml} / \mathrm{min} \times$

$60 \mathrm{~min} / 10 \mathrm{~cm}=$ amount $/ \mathrm{cm}$ intestine $/$ hour .

The study was approved by the Ethical Committee of the Medical Faculty, Uppsala University. Patients and controls gave their informed consent to participation in the study.

\section{Results}

The concentrations of beta ${ }_{2}$-microglobulin (beta ${ }_{2}$-micro), albumin, and hyaluronan in jejunal perfusion fluid from patients with coeliac disease and controls are given in Table II. Under basal conditions, the albumin concentrations were similar in patients and controls. Increased concentrations of hyaluronan were observed in the patient group but the difference did not reach statistical significance. The beta ${ }_{2}$-micro concentrations were on average trebled in the coeliacs compared with the controls $(\mathrm{p}<0.01)$. The basal appearance rates of the measured substances

TABLE II The concentrations ${ }^{\star}$ of beta ${ }_{2}$-microglobulin, albumin, and hyaluronan in jejunal perfusion fluid from patients with coeliac disease and healthy controls and the calculated corresponding jejunal appearance rates

\begin{tabular}{|c|c|c|c|c|c|c|}
\hline \multirow[b]{2}{*}{ Group } & \multicolumn{2}{|l|}{ Beta $_{2}-$ micro } & \multicolumn{2}{|c|}{ Albumin } & \multicolumn{2}{|c|}{ Hyaluronan } \\
\hline & $m g / l$ & $\mu \mathrm{g} / \mathrm{cm} / \mathrm{h}$ & $m g / l$ & $\mu \mathrm{g} / \mathrm{cm} / \mathrm{h}$ & $\mu g / l$ & $\mathrm{ng} / \mathrm{cm} / \mathrm{h}$ \\
\hline $\begin{array}{l}\text { Coeliac disease } \\
(n=10) \\
\text { Controls }(n=14)\end{array}$ & $\begin{array}{l}0.18(0.04) \dagger \\
0.05(0.01)\end{array}$ & $\begin{array}{l}3 \cdot 2(0 \cdot 8) \dagger \\
0 \cdot 86(0 \cdot 2)\end{array}$ & $\begin{array}{l}31(5) \\
28(6)\end{array}$ & $\begin{array}{l}565(102) \\
512(114)\end{array}$ & $\begin{array}{l}83(35) \\
34(4)\end{array}$ & $\begin{array}{r}1488(627) \\
613(75)\end{array}$ \\
\hline
\end{tabular}

${ }^{\star}$ Means (SEM); Statistical differences between patients and controls are indicated $\nmid p<0 \cdot 01$
TABLE III The jejunal secretion rates of beta ${ }_{2}-$ micro, $_{\text {, }}$ albumin and hyaluronan in patients with coeliac disease, subgrouped into patients with normal small bowel mucosa and patients with active disease as evidenced by villous abnormalities in small bowel biopsies

\begin{tabular}{lllc}
\hline Group & $\begin{array}{l}\text { Beta } 2 \text {-micro } \\
\mu \mathrm{g} / \mathrm{cm} / \mathrm{h}\end{array}$ & $\begin{array}{l}\text { Albumin } \\
\mathrm{\mu g} / \mathrm{cm} / \mathrm{h}\end{array}$ & $\begin{array}{l}\text { Hyaluronan } \\
\mathrm{ng} / \mathrm{cm} / \mathrm{h}\end{array}$ \\
\hline $\begin{array}{l}\text { Non-active disease }(\mathrm{n}=3) \\
\text { Active disease }(\mathrm{n}=8)\end{array}$ & $\begin{array}{l}1 \cdot 8(0 \cdot 3) \\
3 \cdot 4(0.9)\end{array}$ & $\begin{array}{l}285(89) \\
625(99)\end{array}$ & $\begin{array}{c}641(59) \\
1709(771)\end{array}$ \\
\hline
\end{tabular}

Means (SEM)

were calculated (Table II). The differences observed between the groups were not influenced by this calculation. Those patients who had active coeliac disease defined by histological findings on biopsy presented higher appearance rates of all substances, but the differences did not reach statistical significance (Table III).

The serum concentrations of albumin and hyaluronan in the coeliac group were $43(0.7)$ (SEM) $\mathrm{g} / \mathrm{l}$ and $44 \cdot 5(8 \cdot 8) \mu \mathrm{g} / \mathrm{l}$, respectively, and within the reference values at our laboratory; the normal range for albumin being $36-48 \mathrm{~g} / \mathrm{l}$ and for HA $10-100 \mu \mathrm{g} / \mathrm{l}$. The serum beta 2 -micro values tended to be slightly increased in the patient group, $1 \cdot 8(0 \cdot 1) \mathrm{mg} / \mathrm{l}$; the reference value at our laboratory is $1 \cdot 1-2 \cdot 1 \mathrm{mg} / \mathrm{l}$. The ratios between the serum and jejunal fluid concentrations in the patients group were for beta $_{2}$-micro $0 \cdot 10$, for albumin $\mathbf{0 . 0 0 0 7}$ and for hyaluronan 1.94.

During challenge with gliadin the appearance rates of beta $_{2}$-micro, albumin and hyaluronan started to increase at the same time and on average 40 minutes after administration of gliadin (Figs 2,3). A tendency towards an earlier onset of the influx of beta ${ }_{2}$-micro was observed. The peak appearance rates of the measured variables were on average twice as high, compared with basal appearance rates, and reached a maximum 60 minutes after gliadin administration. The enhanced appearance rates of hyaluronan and albumin levelled off after that time but remained raised during the observation period of 120 minutes after the gliadin challenge. In contrast, the appearance rate of beta ${ }_{2}$-micro started to decrease after $60 \mathrm{~min}$ and returned to basal levels within the $120 \mathrm{~min}$ observation period (Fig 2). Gliadin challenge tests of healthy controls did not alter the appearance rates of the measured substances (data not shown). Among the coeliac patients the maximum appearance rates varied within narrow limits (Fig 4). The maximum increases, in absolute or relative terms, were apparently not related to differences in the histopathological activity of the disease.

Only two of the patients had minor subjective reactions during gliadin challenge. One reported a burning pain that he claimed to be the same as after accidental gluten ingestion. The other patient reported nausea. The other five patients reported no reactions. None of the controls complained of any reactions.

\section{Discussion}

The validity of the present investigation rests upon the quality of the perfusions. Previous investigations of small bowel secretion have mainly been performed by open perfusion systems or aspiration. The advantage of a 


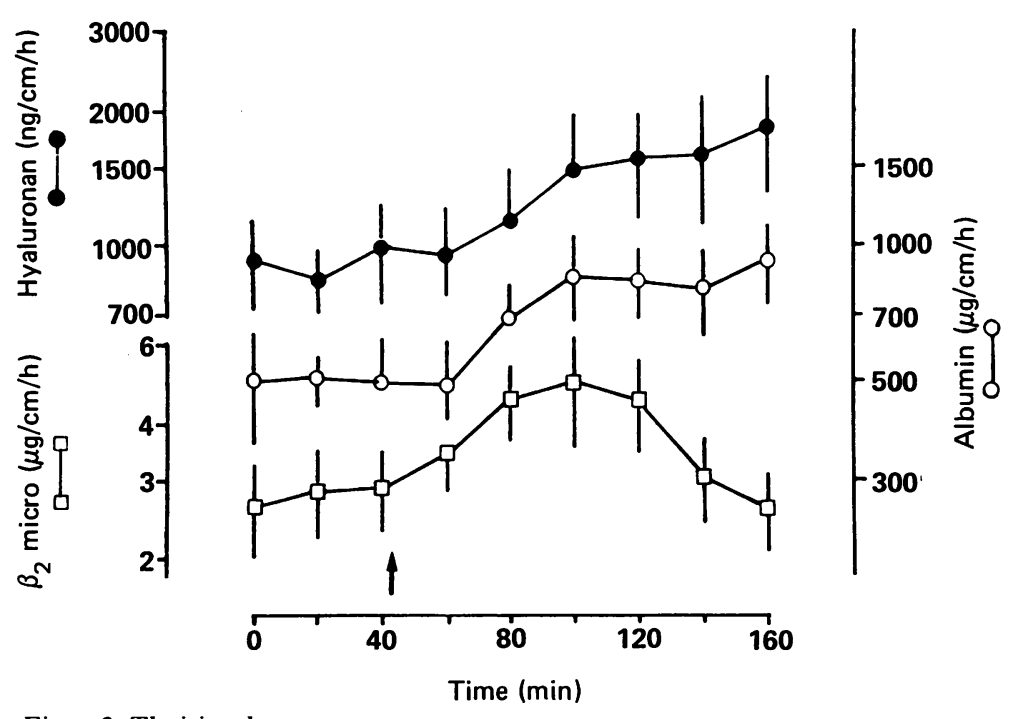

Figure 2: The jejunal appearance rates of beta ${ }_{2}$ microglobulin, albumin and hyaluronan in patients $(\boldsymbol{n}=$ 7) with coeliac disease before and after gliadin challenge of the perfused jejunal segment. Each data point represents the mean value (SEM). The arrow indicates when gliadin administration was started.

Figure 3: The individual maximum appearance rates of hyaluronan, albumin and beta $a_{2}$-microglobulin in seven patients with coeliac disease under basal conditions $(A)$ and after gliadin challenge of the perfused jejunal segment $(B)$. The increases of the appearance rates, basal to maximal, were statistically significant; beta $a_{2}$ micro $(p<0.02)$, albumin $(p<0.02)$, and hyaluronan $(p<0.05)$. The maximal appearance rates were reached 40-120 min after gliadin challenge was started.

Hyaluronan

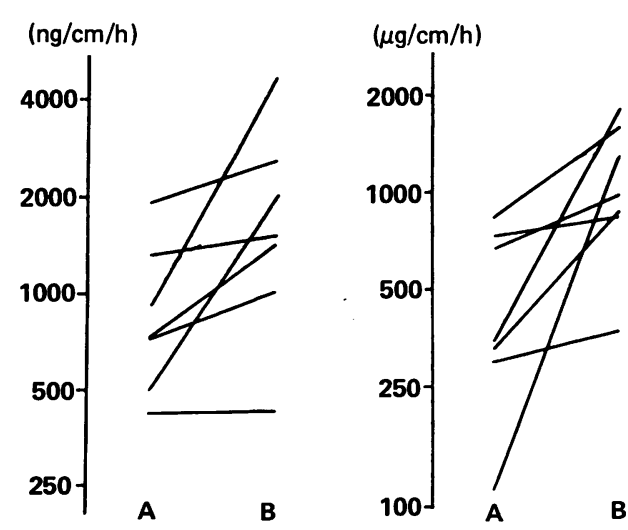

proximal occluding balloon in order to minimise contamination of secretions by acid, bile, and pancreatic juice has been recognised. ${ }^{18}{ }^{19}$ With the present technique, the proximal leakage as tested by phenol red was minimal. In order to avoid possible influence of minute amounts of proteolytic enzymes on the measurements, we included a number of precautions including the addition of a trypsin inhibitor to the perfusion fluid, collection of fluid on an ice bath and addition of a serine protease inhibitor to the samples before assay. The advantages of a closed segment perfusion with a distal occluding balloon are obvious during local challenge. This allows an excellent recovery of infused perfusion fluid and prevents a distal reflux of fluid which may become considerable and unpredictable. ${ }^{20} \mathrm{~A}$ consequence of such an influence is that a longer segment than estimated is in fact studied, leading to underestimation of the secretion. ${ }^{19}$ In the present study the recovery of the perfusion fluid was quite acceptable, as judged by the recovery of the ${ }^{14} \mathrm{C}$-PEG 4000 marker in the collected perfusion fluid.

By the use of our perfusion system we have shown that challenge of a defined jejunal segment with gliadin induces a two-fold increase of the jejunal appearance rates of beta microglobulin, albumin, and hyaluronan in patients with coeliac disease but not in healthy controls. The parallel increase of the appearance rates of beta ${ }_{2}$-micro, albumin, and hyaluronan after gliadin administration should favour the
B

Albumin

$$
\beta_{2} \text { micro }
$$

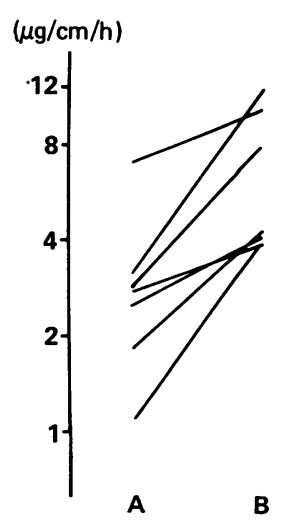

hypothesis that the altered appearance rates of these substances reflect a common mechanism. It is reasonable to attribute this reaction of the jejunum to a gliadin induced inflammatory damage of the mucosa, because infiltration of inflammatory cells can be seen in jejunal biopsies taken within a few hours after gluten challenge of patients with coeliac disease. ${ }^{46}$

Under basal conditions the measured albumin concentrations in the jejunal perfusion fluids of healthy controls and coeliac patients were about $0 \cdot 1 \%$ of the serum concentrations, reflecting the degree of passive leakage of proteins from the plasma and interstitial fluid compartments to the jejunal fluid. ${ }^{7}$ The basal appearance rate of albumin in the whole group of coeliac patients was similar to that seen in controls. Patients with histologically active coeliac disease, however, presented an increased albumin appearance rate compared with coeliac patients with a normal histology of the mucosa. These findings support previous observations of intestinal albumin loss in patients with active coeliac disease. ${ }^{21}$

The serum concentrations of hyaluronan vary between $10-100 \mu \mathrm{g} / \mathrm{l}$ in healthy subjects, and patients with coeliac disease had similar serum values. The concentrations of hyaluronan measured in the jejunal perfusion fluid are close to hyaluronan concentrations in the blood circulation. Thus, a passive leakage from the plasma compartment cannot explain the high jejunal perfusion fluid concentrations of hyaluronan. A more likely source of hyaluronan is the lymph/ interstitial fluid, as the concentration of hyaluronan is about 100 times higher in lymph fluid than in plasma. ${ }^{9}$ In contrast the albumin concentrations in lymph fluid are close to the serum concentrations of albumin. ${ }^{9}$ Provided that the appearance of hyaluronan and albumin in the jejunal fluid reflects a common mechanism, both substances should then be secreted into the intestinal lumen mainly from the lymph/ interstitial fluid compartment and to a lesser extent from the plasma compartment. This would mean that the increase of the appearance rate of albumin and hyaluronan observed after gliadin challenge should reflect a lymph/ interstitial fluid oedema with enhanced leakage of interstitial constituents. The main synthesis of hyaluronan is brought about by mesenchymal cells of the connective tissue. ${ }^{8}$ The demonstration in healthy individuals of a considerable hyaluronan appearance into the jejunal lumen might therefore reflect the normal rapid turnover of the intestinal mucosa. The increased basal appearance rate of hyaluronan observed in active coeliac disease may partly be the result of an enhanced local hyaluronan synthesis caused by stimulated cell replication of the mucosa. ${ }^{22}{ }^{23} \mathrm{It}$ seems less likely, however, that the increase of hyaluronan observed as early as $\mathbf{4 0}$ minutes after gliadin challenge should be caused by the induction of hyaluronan synthesis.

Previously, Blanco et al have reported increased serum concentrations of beta $\mathrm{b}_{2}$-micro in coeliac disease, ${ }^{24}$ a finding we were able to confirm in our patients. Furthermore, we observed a three-fold higher basal jejunal appearance rate of beta $\mathbf{b}_{2}$-micro. Based on these findings in intestinal fluid, it is reasonable to 
propose that the increased serum concentrations of beta ${ }_{2}$-micro in coeliac disease might at least partly reflect an enhanced production of this protein in the intestine. All nucleated cells are able to synthesise beta $a_{2}$-micro. An increased production of beta $a_{2}$-micro in the mucosa may reflect the increased enterocyte turnover which has been documented to be greatly enhanced in coeliac disease." An alternative explanation could be an increased production from invading cells, mainly lymphocytes. ${ }^{23}$ Beta ${ }_{2}$-microroglobulin is a low molecular weight substance (Mw 11800 daltons) and should appear more easily than albumin in the intestinal fluid. It was also calculated that the ratio between the concentration of beta ${ }_{2}$-micro in jejunal fluid in relation to serum levels was relatively higher in healthy controls than the corresponding ratio for albumin. The appearance rate of beta ${ }_{2}$-micro was on average doubled after gliadin challenge of coeliac patients. The increased influx of beta ${ }_{2}-$ micro tended to precede the increased influxes of albumin and hyaluronan. Furthermore, in contrast with these substances, the enhanced appearance of beta $a_{2}$-micro was transient and at the end of the 120 min follow up period after gliadin administration, its appearance rate had returned to basal levels. These differences in the behaviour of the measured variables can possibly be explained if we assume that the leakage from the tissue is a transient phenomenon in our gliadin challenge model. In such a situation, differences in molecular sizes of the leaking substances might influence the kinetics of their transport to the jejunal lumen. Alternatively the rapid increase and decrease of beta -micro could $^{-}$ reflect the local tissue damage elicited by the gliadin challenge. Enterocyte damage has been reported to occur within two hours. ${ }^{+6}$

In conclusion, we have shown that a very transient challenge of the small bowel with gliadin induces an increase in the appearance rates of beta ${ }_{2}$-micro, albumin, and hyaluronan in patients with coeliac disease but not in healthy controls. The appearance of these substances in increased amounts in the jejunal fluid points to a gliadin induced lymphoedema and an enhanced leakage fromt he interstitial/lymph fluid. The perfusion technique used allows kinetic studies of the local intestinal reactions mediated by gliadin and it restricts the gliadin challenge in dose, time, and intestinal area.

This work was supported by grants from the Swedish Medical Research Council, the Swedish Life Insurance Companies' Trust for Medical Research, and Pharmacia AB, Sweden. The skilful assistance of Carina Nimbratt, Eva Sabler, and Cathrine Edström is gratefully acknowledged.

1 Booth CC. Enterocvte in coeliac disease. $B r$ Med $\mathcal{F} 1970 ; 3$ $725-32,1970 ; 4: 1+7$

2 Marsh $\mathrm{MN}$, Hinde J. Inflammatory component of coeliac sprue mucosa. I. Mast cells, basophils and eosinophils. Gastroenterologv 1985; 89: 92-101

3 Marsh MN. Studies of the intestinal lymphoid tissue, III Quantitative analysis of epithelial lymphocytes in the small intestine of human control subjects and of patients with coeliac sprue. Gastroenterology 1980; 79: 481-92.

4 Anand BS, Piris J, Jerrome DW, Offord RE, Truelove SC The timing of histological damage following a single challenge with gluten in treated coeliac disease. $Q \mathcal{F} M e d$ $1981 ; 197: 83-94$.

5 Marsh MN. Immunocytes, enterocytes and the lamin propria, an immunopathological framework of coeliac disease. F R Coll Phys 1983; 197: 205-12.

6 Freedman AR, McCarthy JC, Nelufer JM, Ciclitira PJ Timing of infiltration of $\mathrm{T}$ lymphocytes induced by gluten Timing of infiltration of T lymphocytes induced by gluten 40: $741-5$.

7 Knutson L, Odlind B, Hällgren R. A nem technique for segmental jejunal perfusion in man. Am $\mathcal{F}$ Gastroenterol 1989 (In press)

8 Laurent ' $\Gamma$ C. Structure, function and turn-over of the extracellular matrix. In: Staub NC, Hogg JC, Hargen AR, eds Intestinal lymphatic liquid and solute movement. Adv Microcirc 1987; 13: 15-34.

9 Tengblad A, Laurent U, Lilja K, et al. Concentration and relative molecular mass of hyaluronate in lymph and blood. Biochem F 1986; 236: 521-5.

10 Bernier GM, Fanger MW. Synthesis of beta2-microglobulin by stimulated lymphocytes. F Immunol 1972; 109: 407-9.

11 Simpson FG, Howdle PD, Robertson DAF, Losowsky MS Jejunal biopsy and lymphocyte co-culture in coeliac disease. Jejunal biopsy and lymphocyte co-culture

12 Stern AL, Hogan DL, Isenberg JI. A new method for quantification of ion fluxes across the in vivo human gastric mucosa Gastroenterology 1984; 86: 60-70.

13 George JD. New clinical method for measuring the rate of gastric emptying; the double sampling test meal. Gut 1968; 9: $237-42$

14 Rick W. Trypsin. In: Bergmayer HU, ed. Methods of enzymatic analyses. Vol 2. 2nd ed. New York: Academic enzymatic analyses.
Press, 1974: 1013-24.

15 Kunitz M. Crystalline soybean trypsin inhibitor II. General properties. $\tilde{f}$ Gen Physiol 1947; 30: 291.

16 Laurent UBG, Tengblad A. Determination of hyaluronate in biological samples by a specific radioassay technique. Ann Biochem 1980; 109: 396-94.

17 Engström-Laurent A. Laurent UBG, Laurent TC. Concentration of sodium hyaluronate in serum. Scand 7 Clin Lab Invest 1985; 45: 497-504

18 Sladen GE, Dawson AM. Further studies on the perfusion method for measuring intestinal absorption in man, the effects of a proximal balloon and a mixing segment. Gut 1970; 11: $947-54$

19 Modigliani R, Bernier JJ. Absorption of glucose, sodium and water by the human jejunum studied by intestinal perfusion with a proximal balloon and at variable flow rates. Gut 1971 12: 184-93.

20 Soergel $\mathrm{KH}$. An evaluation of perfusion techniques in the study of water and electrolyte absorption in man. Gut 1969; 10: 601-2.

21 Jarnum S, Jensen KB, Soltoft J, Westergaard H. Protein loss and turnover of albumin, IgG and IgM in adult coeliac disease. In: Booth CC, Dowling RH, eds. Coelia
Edinburgh: Churchill Livingstone, 1970: 163-72.

22 Watson AJ, Wright NA. Morphology and cell kinetics of the jejunal mucosa in untreated patients. Clin Gastroenterol 1974; 3: 11-31

23 Marsh MN, Haeney MR. Studies of intestinal lymphoid tissue, VI. Proliferative response of small intestinal epithelial lymphocytes distinguishes gluten from non-gluten induced enteropathy. $\mathcal{F}$ Clin Pathol 1983; 36: 149-60.

24 Blanco A, Alonso M, Cilleruelo ML, Solis P, Calvo C, Sanche\% Villares $\mathrm{E}$. Increased serum beta2-mircoglobulin levels in active celiac disease. I Pediatr Gastroenterol Nutr 1985; 4: 388-92. 